\title{
Visual Respiratory Feedback in Virtual Reality Exposure Therapy: A Pilot Study
}

\author{
Deniz Mevlevioğlu \\ d.mevlevioglu@cs.ucc.ie \\ University College Cork \\ Cork, Ireland
}

\author{
David Murphy \\ d.murphy@cs.ucc.ie \\ University College Cork \\ Cork, Ireland
}

\author{
Sabin Tabirca \\ tabirca@cs.ucc.ie \\ University College Cork \\ Cork, Ireland
}

\begin{abstract}
As the use of Virtual Reality (VR) expands across fields, new kinds of interaction methods are introduced. This study presents the Visual Heights VR experience that integrates natural breathing as an input method to provide visual respiratory feedback. Incorporating spatial audio, haptic feedback and breath visualisation, the experience aims to be highly immersive. This experience was made to be used as part of a controlled pilot study to see the effect of respiratory feedback on the user's anxiety levels. The user's anxiety is assessed by their heart rate, brain electrical activity, skin conductance and respiratory rate. These biosignals are recorded within the experience; captured by external hardware. The pieces of hardware used were Galvanic Skin Response to measure skin conductance, photoplethysmogram to measure heart rate; Electroencephalogram to measure the electrical activity in the brain, and a prototype device that records airflow on an axis from -1 to 1 for respiratory rate. It was found that the aforementioned prototype was not sufficient for calculating the respiratory rate. Results of the controlled study showed that the Visual Heights VR experience delivered the expected positive correlation between skin conductance and perceived height $(\mathrm{r}=.491, \mathrm{p}<.05, \mathrm{~N}=1543)$ which suggests it is plausible to be used as a material for further research. As the integration of user's physiological signals and breathing for visual feedback can contribute to therapeutic uses of VR, research with bigger sample sizes will be conducted to better investigate the relationship between visual respiratory feedback and anxiety using the Visual Heights VR experience.
\end{abstract}

\section{CCS CONCEPTS}

- Human-centered computing $\rightarrow$ Human computer interaction (HCI); Interaction design; Interaction techniques; Virtual reality.

\section{KEYWORDS}

biosensors, biosignals, VR, exposure therapy, anxiety, GSR, PPG, EEG

ACM Reference Format:

Deniz Mevlevioğlu, David Murphy, and Sabin Tabirca. 2021. Visual Respiratory Feedback in Virtual Reality Exposure Therapy: A Pilot Study. In $A C M$

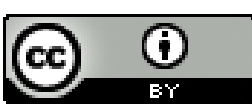

This work is licensed under a Creative Commons Attribution International 4.0 License. IMX '21, June 21-23, 2021, Virtual Event, NY, USA

(C) 2021 Copyright held by the owner/author(s).

ACM ISBN 978-1-4503-8389-9/21/06.

https://doi.org/10.1145/3452918.3458799
International Conference on Interactive Media Experiences (IMX '21), June 21-23, 2021, Virtual Event, NY, USA. ACM, New York, NY, USA, 6 pages. https://doi.org/10.1145/3452918.3458799

\section{INTRODUCTION}

A phobia is an anxiety disorder characterised by the illogical fear of an object or a situation that interferes with the person's daily life [5]. One of the leading treatment methods for it is called graded exposure therapy or systematic desensitisation [4]. Graded exposure therapy works by coupling gradual exposure to the fear-inducing object or situation and relaxation techniques to reduce the association between fear and the feared stimulus [14].

Virtual Reality Exposure Therapy (VRET) administers exposure therapy via a virtual simulation using a 3-dimensional display device called a head-mounted display (HMD). Since the first successful case study conducted by Rothbaum in 1995 [24], VRET has been becoming more and more popular [23, 31]. In a virtual environment, it is really easy to control the environment and the exposure and adjust it along the way based on the patient's needs. This can be used to tailor the experience to suit the patient's needs to increase effectiveness.

This study is a pilot study to inform a larger study on the usability of the materials. The main study sets out to integrate natural breathing into a Virtual Reality Environment (VRE) by providing visual feedback on respiration. It is an environment to be used as part of VRET, so it aims to be highly immersive and deliver the natural reaction to height. The experiment investigates whether the environment delivers this reaction.

The experience sets out to be highly immersive with the integration of natural elements into the virtual environment. Most of the experience takes place inside an elevator, and the user is virtually taken to different heights. The immersion is increased by the use of ambisonic spatial audio, haptic feedback using a vibrotactile plate to imitate the vibration of an elevator, and visual feedback on height and respiration.

The study involves the development of such an experience that would provide feedback on the user's breath reliably and record relevant physiological information from the user that would be useful in measuring anxiety. The physiological data is multimodal and includes heart rate, skin conductance, electrical brain activity and respiratory rate. These were collected using Bluetooth on-body sensors without limiting the user's mobility.

\section{RELATED WORK}

The current study extends upon two prior studies; the first conducted by Rachel Barry in 2017 [25] and the second by Sean Kearney in 2018 [11]. Kearney's project is based on Barry's project, it builds 
an immersive experience where the environment is controlled to imitate symptoms of a panic attack and logs physiological information from the user.

The experiment will also be used as an opportunity to see if the respiratory rate recorded by the breath controller prototype would be a reliable measure of anxiety by seeing the correlation between respiratory rate and skin conductance. This would be particularly useful in future experiences if such a device can be used for both assessing anxiety and as a controller.

\subsection{Virtual Reality Exposure Therapy}

There have been many studies assessing the effectiveness of VRET in treating different types of anxiety disorders [17, 18, 29]. While the effectiveness of each system varies, the meta-analysis conducted by Opris et al. in 2012 [18] over 23 studies with a total of 608 subjects, found that participants who received VRET showed a lot more improvement compared to a wait-list control group and similar results to the group who received traditional exposure therapy. The improvement was also long-lasting and increased the quality of life for the participants. This evidence strongly points towards VRET being a viable treatment for anxiety disorders.

In a study by Schuemie et al. [26], it was discovered that the VR experience was successful in delivering the effect of a stressor. Additionally, virtual height correlated with elevated levels of anxiety in users with or without acrophobia. This is presumably due to heights innately being assessed as dangerous by humans, however, acrophobia would be defined as an irrational fear of heights beyond the average person's fear [4].

\subsection{Respiratory Feedback for Relaxation and Breath Integration in Virtual Reality}

Breathing exercises have long been used in combination with exposure therapy to induce relaxation. Breathing exercises can be used for stress-relief, pain-relief and meditation [8]. One breathing technique used in exposure therapy is progressive muscle relaxation, which includes manipulation of the person's respiration to encourage relaxation [2].

In a study by Philippot et al. in 2002 [20], it was shown that it is possible to manipulate a person's emotions using breathing patterns and feedback by presenting them with breathing instructions. A study by Zeier in 1984 [32] which focused on biofeedback for respiratory meditation such as music and relaxing sentences achieved greater arousal reduction when compared to the control group that received no feedback.

There have been many games and experiences that incorporate bio-feedback mechanisms in a way to enhance health and provide therapy $[1,7,15]$. Implementation of respiratory feedback as part of games led to applications such as "Chill-Out" [19] or "BreatheVR" [16]. In both of these applications, the goal to lower arousal levels during stressful stimuli was achieved. BreatheVR was used as part of a treatment for chronic pain and generalised anxiety disorder. Chillfish by Sonne and Jensen [27] offers breathing exercises to help children with ADHD control their stress levels.

BreathingVR [12] by Breathing Labs uses a respiratory belt to provide a breathing exercise for two and a half minutes that visualises their lungs as they breathe in and out while Deep by Harris and Smit [9] offers a meditative VR experience with the presence of respiratory feedback.

\section{EXPERIENCE DESIGN AND IMPLEMENTATION}

The Experience includes two scenes which we will call elevator scene and nature scene. Nature scene is the initial scene and it allows for the user to get used to the environment and receive instructions. It also serves as a safe space for the user to fall back to in the case that they feel too stressed. It includes an open field with trees, grass and flowers and flowers move based on the user's breathing (figure 1). The user can return to this scene by clicking a button or automatically if the signal readings match certain criteria.

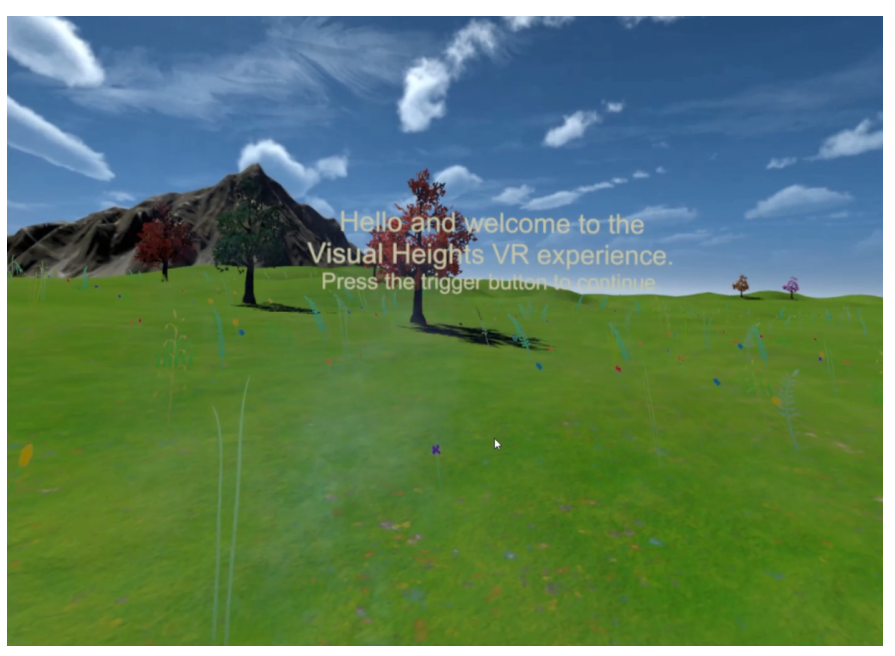

Figure 1: Respiratory Feedback in the form of EBC in Nature Scene

The elevator scene is the scene with the stressing stimulus; visual height. The user is virtually inside a glass elevator outside of a large building in a big city (figure 2). The elevator includes a big elevator button with an indicator light over it. The user can click the elevator button when they are ready to ascend to the next level and experience higher exposure levels. The highest virtual height that the elevator can reach is 66 meters and it takes 6 levels for the user to get there. The user has to spend at least 45 seconds on each level before they can proceed to the next one. When the user has spent at least 45 seconds, there is an auditory cue that is similar to an elevator "ding" sound, as well as a visual cue of the indicator light above turning green from red.

\subsection{Audio and Haptics}

Sound is spatial and recorded in four channels (first-order ambisonics). Elevator sound was recorded in a glass elevator of roughly the same size and speed. The haptic feedback in elevator movement is provided by applying a low-pass filter on the elevator sound and playing through a vibrotactile plate located under the play area of the HMD. The haptic feedback and audio are synced. 


\subsection{Respiratory Feedback}

The main choice of visual feedback for respiration in this experience is Exhaled Breath Condensate (EBC), which refers to vapour arising from the mouth when a person exhales during cold weather (figures 1 and 2). This form of feedback was chosen to avoid distracting the user and increase immersion in the experience. In nature scene, there is additional feedback provided in the form of grass and flowers swaying with the user's exhalation. The breathing is recorded using a prototype that has airflow sensors attached to a headset.

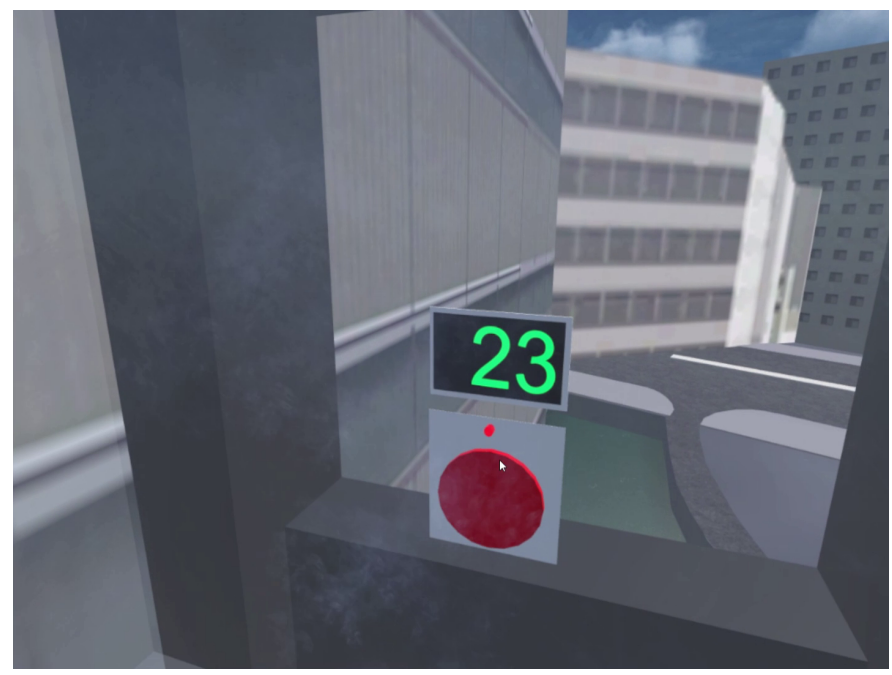

Figure 2: Respiratory Feedback in the Form of EBC in Elevator Scene

\subsection{Recording of Physiological Signals}

Similar to Kearney's study [11], physiological information was collected during the experience and timestamped. The recorded signals are low alpha, high alpha, low beta, high beta, delta, theta, low gamma, high gamma brain wave bands; and beta to alpha ratio, skin conductance, skin resistance, heart rate, respiratory rate and respiratory variance. The reason we chose to examine electrical brain activity is prior research linking high beta and low alpha rates to negative mood and stress [3]. Similarly, higher heart rate and skin conductivity have been associated with increased arousal [22].

Several devices were used to retrieve the aforementioned information. The first of them is the commercial Shimmer device that uses Galvanic Skin Response (GSR) and a photoplethysmogram (PPG) to measure skin conductance and heart rate, respectively. The reason that a PPG device was used to measure heart rate over an electrocardiogram (ECG) is that it allows higher mobility during the experience. The second is MyndPlay BrainBand to measure electroencephalogram (EEG) to record electrical activity in the brain. This device included a single dry node. And the last is a prototype device that measures airflow to determine the respiratory rate [13]. Please refer to Fig. 3 to see how the equipment was laid out. The signals obtained from Shimmer were processed using the Shimmer API, and the signals obtained from the BrainBand were processed using NeuroSky ThinkGear software. Contextual information was also recorded alongside the physiological signals. This information included the virtual height of the player, time spent looking downwards (obtained from the HMD) and experience events such as next level being unlocked, button being clicked and changing back to nature scene (triggered automatically or manually).

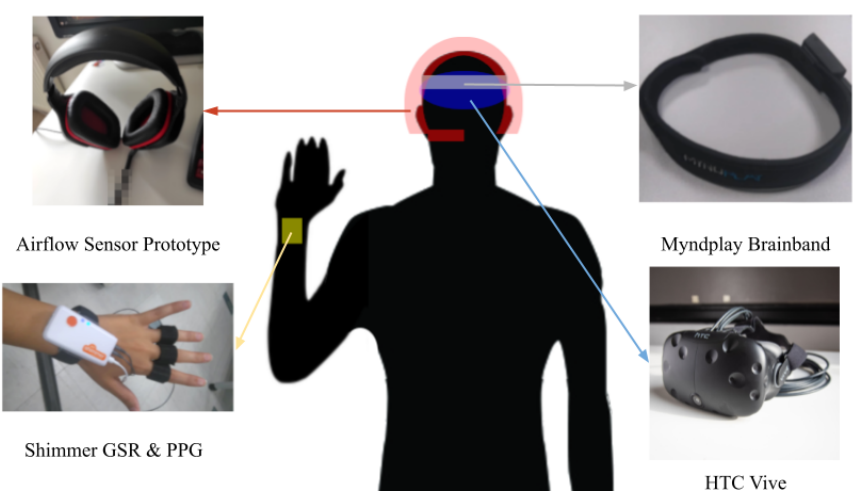

Figure 3: Devices used; Airflow Sensor Prototype, Shimmer, Myndplay Brainband and HTC Vive

\section{RESEARCH METHODS AND RESULTS}

\subsection{Design}

In the experience, nature scene is used to get the users accustomed to VR. The effect of virtual height on arousal is measured by examining the relationship between the two variables with Pearson correlation. Only the information measured while inside the virtual elevator is recorded to minimise the effect of extraneous variables. The anxiety is predicted based on skin conductance and heart rate due to former research linking high heart rate and skin conductance with high arousal [30]. High arousal, in this case, is interpreted as a result of anxiety because that is the natural response of humans to increased height $[4,26]$. However, it is important to note that other causes are possible. Independent Variable (IV) is virtual height and dependent variables (DV) are the skin conductance and heart rate of the user. Extraneous variables are age, exposure to VR, suffering from motion sickness, visual height intolerance (vHI) and presence within VR.

This experiment received ethical approval from the University College Cork Computer Science ethics committee with the code CSIT Ethics 2019-001.

\subsection{Materials}

Visual height intolerance scale (vHISS) was developed by Huppert et al. [10]. It includes two extra questions at the end to determine whether the user suffers from acrophobia. This scale was preferred to other well-established scales such as Acrophobia Questionnaire (AQ) [6] or Heights Interpretation Questionnaire (HIQ) [28] due to being concise and its compatibility with the nature of the study by directly measuring reaction to visual heights in particular. The presence within VR is assessed by Igroup Presence Questionnaire (IPQ) [21]. There were also 5-point Likert type scale questions for 
feedback based on elements which are unique to the experiment (table 1).

The system was tested using the HTC Vive HMD and a computer with a GeForce GTX Titan X graphics card and Intel i7-5820k processor. HTC Vive earphones were used for the audio and haptic feedback was delivered via Clark Synthesis TST329 Gold Transducer placed below the play area, under the floor tiles.

\subsection{Participants}

Eleven participants were drawn from University College Cork students. This was done on a voluntary basis and announced on campus. The mean age was $23.9(\mathrm{~N}=10, \mathrm{SD}=5.28), 4$ out of 11 participants were female. Two participants had never been exposed to VR prior to the experiment, six were only exposed once or twice and three were exposed multiple times. Four participants stated that they experience mild motion sickness while the rest stated that they do not experience motion sickness. Two participants matched the criteria for acrophobia based on vHISS. Seven participants did not experience significant visual height intolerance.

\subsection{Procedure}

Participants were allowed into the VR lab. They first received an information sheet that explained all parts of the experiment and the motives of the study. They were then asked to sign a consent form if they agree to participate. After signing the consent form, they received the first questionnaire, which includes questions about demographics and vHISS. The on-body sensors were placed on the participant by the experimenter before they take part in the VR experience. Utmost care was taken that there were no distractions in the environment during the experience. The experimenter did not provide any additional instructions to the participants apart from the instructions within VR. After the participant was finished with the experience, they received the second questionnaire including questions about the experience and IPQ.

\section{RESULTS AND DISCUSSION}

Every participant was separately analysed for the correlation between their virtual height and skin conductance using Pearson correlation. One participant was an extreme outlier. Upon inspection, it was found that the participant was a poor fit for the GSR sensor due to their finger size. Their information was removed from this analysis because the physiological information recorded from them was unreliable.

There was a positive linear relationship between skin conductance and height across all values $(\mathrm{r}=.491, \mathrm{p}<.05, \mathrm{~N}=1543)$ (figure 4). This relationship was stronger when each participant was evaluated separately $(\mathrm{M}=.78, \mathrm{~N}=10)$. This relationship was true both for participants diagnosed with acrophobia $(\mathrm{M}=.83, \mathrm{~N}=2)$ and participants who were not $(\mathrm{M}=.77, \mathrm{~N}=8)$.

The skin conductance and heart rate values across all participants had a weak positive correlation $(\mathrm{r}=.29, \mathrm{p}<.05, \mathrm{~N}=2391)$. There was no correlation between the arousal level detected from EEG values and either skin conductance $(\mathrm{r}=.01, \mathrm{p}>.05, \mathrm{~N}=3559)$ or heart rate $(\mathrm{r}=.03, \mathrm{p}>0.5, \mathrm{~N}=2389)$.

See table 1 for the responses to the Likert-scale questions presented in the questionnaire administered after the experiment.

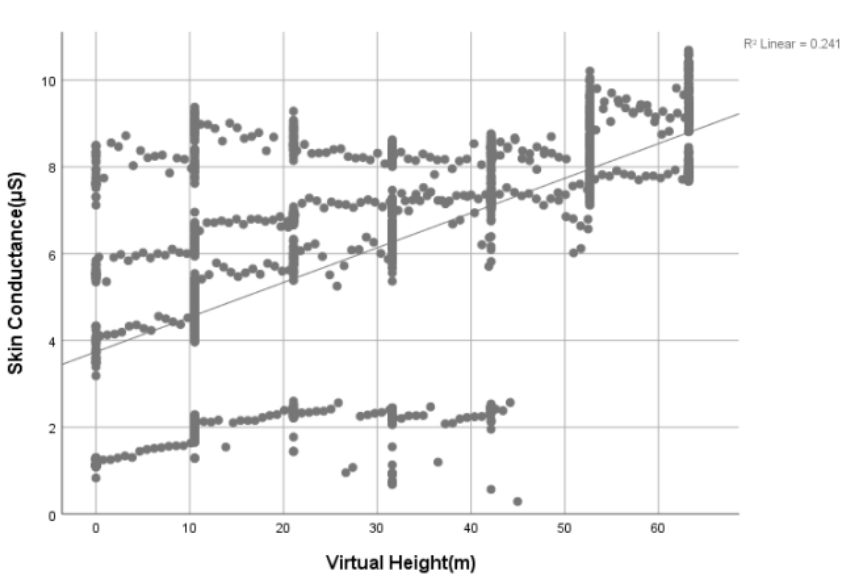

Figure 4: Simple Scatter with Fit Line of Skin Conductance by Virtual Height

Table 1: Summary of the Questionnaire Responses

\begin{tabular}{|l|c|c|c|}
\hline Question & N & Mean & SD \\
\hline $\begin{array}{l}\text { I found the equipment worn during the ex- } \\
\text { periment cumbersome and uncomfortable. }\end{array}$ & 11 & 2.82 & 1.08 \\
\hline $\begin{array}{l}\text { I was aware at all times of the biometric } \\
\text { devices. }\end{array}$ & 11 & 2.73 & 1.10 \\
\hline $\begin{array}{l}\text { I felt motion sickness due to the experi- } \\
\text { ence. }\end{array}$ & 11 & 1.45 & .52 \\
\hline $\begin{array}{l}\text { Visualisation of my breath made me feel } \\
\text { more present in the experience. }\end{array}$ & 4 & 3.25 & .96 \\
\hline $\begin{array}{l}\text { The haptic speakers made me feel more } \\
\text { present in the experience. }\end{array}$ & 11 & 4.18 & .75 \\
\hline I experienced stress in the virtual world. & 11 & 2.45 & 1.44 \\
\hline $\begin{array}{l}\text { I experienced discomfort in the virtual } \\
\text { world. }\end{array}$ & 11 & 2.27 & 1.49 \\
\hline $\begin{array}{l}\text { Amount of equipment I was wearing re- } \\
\text { duced my feeling of presence in the virtual } \\
\text { world. }\end{array}$ & 11 & 2.82 & 1.08 \\
\hline
\end{tabular}

The correlation between heart rate and skin conductance is in the expected direction, and the reason it is not stronger is due to different physiological reactions of different persons to stress and heart rate calculation not being as reliable as skin conductance due to the number of missing values, and incorrect spikes at times due to noise caused by contact issues and movement. The decision not to include heart rate in the correlation was based on the information having too much noise for some participants and skin conductance being an overall more reliable measure of high arousal [30]. The reason there is no correlation between the EEG values and the other two measures might be due to it being more susceptible to noise due to the use of a single dry node. The node was also placed right under the HMD, so it can be affected by the movement of the device 
and the electrical signals. Shimmer GSR was found to be the most reliable method for assessing anxiety out of GSR, PPG and EEG.

The airflow prototype as a way of recording natural breathing was not viable due to two reasons. First was that it was too bulky for use with the HMD; it was moved when the user was trying to move the HMD, which occasionally caused it to slip away from its optimal position. The other reason was the fact that the airflow sensors were quite large and might constrict the breathing of the user when used in conjunction with the HMD. This was deemed potentially distressing and uncomfortable. Even though the device was deemed not optimal for recording respiratory rate, it might be a viable option for conscious use as a controller when the user is aware that their respiration needs to be recorded which will allow them to move the device to get it in optimal placement for both the recording and comfort of breathing.

The experience was shown to be sufficient to create the expected effect of increased skin conductance as the visual height increases. Based on questionnaire responses (table 1), haptic feedback was very successful in increasing the sense of presence. The users have expressed some discomfort and stress within the virtual world. They indicated that the amount of on-body sensors worn had some effect on decreasing immersion and causing discomfort. Users who received visual respiratory feedback said it increased their presence within the experience, but not as much as the haptic speakers. It should be taken into account that these data are limited by being self-report and being asked directly instead of measuring.

The study has several potential limitations. It was conducted under time constraints that made it harder to reach a large and random sample size. The sample may not represent the general population accurately due to participants being students with high technology literacy. Furthermore, detected higher arousal levels were interpreted to be as a result of anxiety from the increased level of heights based on prior research. However, there might be different causes for the increased arousal.

As a pilot study, the study shows that the environment used for the delivery of the stimulus is appropriate. However, it was found that the airflow prototype in its current form was not the right medium for recording respiratory rate or providing respiratory feedback. It is necessary to find a way to use it in conjunction with the HMD without obstructing the user. A respiratory belt or Electrocardiogram (ECG) could be a replacement for the device for further experimentation as they might provide better mobility and accuracy. Additionally, a single dry node was used for EEG for comfort, however, it might be beneficial to use a wet node to reduce noise. Additionally, behavioural information such as head movement recorded from HMD can be used in conjunction with the physiological information to make the results more interpretable. Continuation of this research will incorporate these changes and compare arousal levels between users that receive visual respiratory feedback and a control group.

\section{CONCLUSION}

VR applications are getting more and more common. Past research has shown that the therapeutic use of VR is invaluable $[17,18]$. The authors are researching a system that incorporates natural respiratory feedback into VRET to increase immersion and encourage relaxation. The current pilot study explores the usability of the materials used by this system. The correlation between arousal and virtual height shows that the developed VR experience is capable of producing the wanted effect. However, the device that measures breathing need to be replaced.

More research into emotion detection using physiological responses and the integration of extra senses can contribute to VR therapy methods by providing objective feedback and increasing immersion. It can also contribute to the entertainment value of VR by increasing immersion and tailoring experiences to the users. For example, it can be used in horror games to adjust the horror elements based on the user and make sure they are engaged at all times.

\section{ACKNOWLEDGMENTS}

This publication has emanated from research supported in part by a Grant from Science Foundation Ireland under Grant number $18 / \mathrm{CRT} / 6222$.

\section{REFERENCES}

[1] E. Bisson, B. Contant, H. Sveistrup, and Y. Lajoie. 2007. Functional Balance and Dual-Task Reaction Times in Older Adults Are Improved by Virtual Reality and Biofeedback Training. CyberPsychology \& Behavior 10, 1 (2007), 16-23. https://doi.org/10.1089/cpb.2006.9997

[2] Thomas Budzynski, Johann Stoyva, and Charles Adler. 1970. Feedback-induced muscle relaxation: Application to tension headache. Fournal of Behavior Therapy and Experimental Psychiatry 1, 3 (1970), 205-211. https://doi.org/10.1016/00057916(70) 90004-2

[3] Guillaume Chanel, Julien Kronegg, Didier Grandjean, and Thierry Pun. 2006. Emotion Assessment: Arousal Evaluation Using EEG's and Peripheral Physiological Signals. In Multimedia Content Representation, Classification and Security, Bilge Gunsel, Anil K. Jain, A. Murat Tekalp, and Bülent Sankur (Eds.). Springer Berlin Heidelberg, Berlin, Heidelberg, 530-537. https://doi.org/10.1007/11848035_70

[4] Carlos M. Coelho and Guy Wallis. 2010. Deconstructing acrophobia: physiological and psychological precursors to developing a fear of heights. Depression and Anxiety 27, 9 (2010), 864-870. https://doi.org/10.1002/da.20698

[5] Carlos M. Coelho, Allison M. Waters, Trevor J. Hine, and Guy Wallis. 2009. The use of virtual reality in acrophobia research and treatment. Fournal of Anxiety Disorders 23, 5 (2009), 563-574. https://doi.org/10.1016/j.janxdis.2009.01.014

[6] David Chestney Cohen. 1977. Comparison of self-report and overt-behavioral procedures for assessing acrophobia. Behavior Therapy 8, 1 (1977), 17-23. https://doi.org/10.1016/s0005-7894(77) 80116-0

[7] Diarmaid Fitzgerald, John Foody, Dan Kelly, Tomas Ward, Charles Markham, John Mcdonald, and Brian Caulfield. 2007. Development of a wearable motion capture suit and virtual reality biofeedback system for the instruction and analysis of sports rehabilitation exercises. 2007 29th Annual International Conference of the IEEE Engineering in Medicine and Biology Society (2007). https://doi.org/10.1109/ iembs.2007.4353431

[8] M Franck, H Schäfer, W Stiels, R Wassermann, and JM Herrmann. 1994. Relaxation therapy with respiratory feedback in patients with essential hypertension. Psychotherapie, Psychosomatik, Medizinische Psychologie 44, 9-10 (1994), 316-322. https://doi.org/10.1038/jhh.2008.65

[9] Owen Harris and Niki Smit. [n.d.]. Deep is a meditative game controlled by breathing. http://www.exploredeep.com/. Accessed: 2019-09-30.

[10] Doreen Huppert, Eva Grill, and Thomas Brandt. 2017. A new questionnaire for estimating the severity of visual height intolerance and acrophobia by a metric interval scale. Frontiers in neurology 8 (2017), 211. https://doi.org/10.3389/fneur. 2017.00211

[11] Sean Kearney. 2018. Investigate the use of Galvanic Skin Response, brain-computer interface and heart rate in virtual reality. Master's thesis. University College Cork. unpublished thesis.

[12] Breathing Labs. [n.d.]. Breathing VR: Breathing Virtual Reality Apps for use in psychiatry and rehabilitation. https:/www.breathinglabs.com/latestnews/breathing-vr-breathing-virtual-reality-apps-for-use-in-psychiatry-andrehabilitation/. Accessed: 2019-09-30.

[13] Deniz Mevlevioglu. 2019. Visual respiratory feedback in virtual reality: a pilot study. Master's thesis. University College Cork. unpublished thesis.

[14] K. M. Myers. 2006. Different mechanisms of fear extinction dependent on length of time since fear acquisition. Learning \& Memory 13, 2 (Jan. 2006), 216-223. https://doi.org/10.1101/lm.119806 
[15] Lennart Erik Nacke, Michael Kalyn, Calvin Lough, and Regan Lee Mandryk. 2011. Biofeedback game design. Proceedings of the 2011 annual conference on Human factors in computing systems - CHI 11 (2011). https://doi.org/10.1145/1978942. 1978958

[16] Neon. [n.d.]. BreatheVR. https://www.discoverneon.com/breathevr. Accessed: 2019-09-30.

[17] Dan Opdyke, James S Williford, and Max North. 1995. Effectiveness of computergenerated (virtual reality) graded exposure in the treatment of acrophobia. Am 7 psychiatry 1, 152 (1995), 626-28. https://doi.org/10.1176/ajp.152.4.626

[18] David Opriş, Sebastian Pintea, Azucena García-Palacios, Cristina Botella, Ştefan Szamosközi, and Daniel David. 2011. Virtual reality exposure therapy in anxiety disorders: a quantitative meta-analysis. Depression and Anxiety 29, 2 (July 2011), 85-93. https://doi.org/10.1002/da.20910

[19] Avinash Parnandi, Beena Ahmed, Eva Shipp, and Ricardo Gutierrez-Osuna. 2013 Chill-Out: Relaxation training through respiratory biofeedback in a mobile casual game. In International Conference on Mobile Computing, Applications, and Services. Springer, 252-260. https://doi.org/10.1007/978-3-319-05452-0_18

[20] Pierre Philippot, Gaëtane Chapelle, and Sylvie Blairy. 2002. Respiratory feedback in the generation of emotion. Cognition \& Emotion 16, 5 (2002), 605-627. https://doi.org/10.1080/02699930143000392

[21] Holger Regenbrecht and Thomas Schubert. 2002. Real and Illusory Interactions Enhance Presence in Virtual Environments. Presence: Teleoperators and Virtual Environments 11, 4 (2002), 425-434. https://doi.org/10.1162/105474602760204318

[22] Tatyana Reinhardt, Christian Schmahl, Stefan Wüst, and Martin Bohus. 2012 Salivary cortisol, heart rate, electrodermal activity and subjective stress responses to the Mannheim Multicomponent Stress Test (MMST). Psychiatry Research 198, 1 (2012), 106-111. https://doi.org/10.1016/j.psychres.2011.12.009

[23] Giuseppe Riva. 2005. Virtual Reality in Psychotherapy: Review. CyberPsychology \& Behavior 8, 3 (2005), 220-230. https://doi.org/10.1089/cpb.2005.8.220

[24] Barbara Olasov Rothbaum, Larry F. Hodges, Rob Kooper, Dan Opdyke, James S. Williford, and Max North. 1995. Virtual reality graded exposure in the treatment of acrophobia: A case report. Behavior Therapy 26, 3 (1995), 547-554. https: //doi.org/10.1016/s0005-7894(05)80100-5

[25] Vincent Russell, Rachel Barry, and David Murphy. 2018. HAVE Experience: an investigation into VR empathy for panic disorder. In 2018 IEEE Games, Entertainment, Media Conference (GEM). IEEE, 1-9.

[26] MJ Schuemie, M Bruynzeel, L Drost, M Brinckman, G De Haan, PMG Emmelkamp, and CAPG Van der Mast. 2000. Treatment of acrophobia in virtual reality: A pilot study. In Conference Proceedings Euromedia. 271-275. https://doi.org/10.1177/ 1357633X15598243

[27] Tobias Sonne and Mads Møller Jensen. 2016. ChillFish. Proceedings of the TEI 16: Tenth International Conference on Tangible, Embedded, and Embodied Interaction TEI 16 (2016). https://doi.org/10.1145/2839462.2839480

[28] Shari A. Steinman and Bethany A. Teachman. 2011. Cognitive processing and acrophobia: Validating the Heights Interpretation Questionnaire. Fournal of Anxiety Disorders 25, 7 (2011), 896-902. https://doi.org/10.1016/j.janxdis.2011. 05.001

[29] Guang-Xin Wang and Li Li. 2013. Virtual Reality Exposure Therapy of Anxiety Disorders. Advances in Psychological Science 20, 8 (July 2013), 1277-1286. https://doi.org/10.3724/sp.j.1042.2012.01277

[30] Brenda K Wiederhold, Dong P Jang, Sun I Kim, and Mark D Wiederhold. 2002. Physiological monitoring as an objective tool in virtual reality therapy. CyberPsychology \& Behavior 5, 1 (2002), 77-82. https://doi.org/10.1089/ 109493102753685908

[31] Brenda K. Wiederhold and Mark D. Wiederhold. 1998. A Review of Virtual Reality as a Psychotherapeutic Tool. CyberPsychology \& Behavior 1, 1 (1998), 45-52. https://doi.org/10.1089/cpb.1998.1.45

[32] Hans Zeier. 1984. Arousal reduction with biofeedback-supported respiratory meditation. Biofeedback and Self-Regulation 9, 4 (1984), 497-508. https://doi. org/10.1007/bf01000565 